\title{
Toward physiological indices of emotional state driving future ebook interactivity
}

Jan B.F. van Erp, Maarten A. Hogervorst, Ysbrand D. van der Werf

Ebooks of the future may respond to the emotional experience of the reader. (Neuro-) physiological measures could capture a reader's emotional state and use this to enhance the reading experience by adding matching sounds or to change the storyline therewith creating a hybrid art form in between literature and gaming. We describe the theoretical foundation of the emotional and creative brain and review the neurophysiological indices that can be used to drive future ebook interactivity in a real life situation. As a case study, we report the neurophysiological measurements of a bestselling author during nine days of writing which can potentially be used later to compare them to those of the readers. In designated calibration blocks, the artist wrote emotional paragraphs for emotional (IAPS) pictures. Analyses showed that we can reliably distinguish writing blocks from resting but we found no reliable differences related to the emotional content of the writing. The study shows that measurements of EEG, heart rate (variability), skin conductance, facial expression and subjective ratings can be done over several hours a day and for several days in a row. In follow-up phases, we will measure 300 readers with a similar setup. 


\section{Toward physiological indices of emotional state driving future ebook}

2 interactivity

3 Jan B.F. van Erp ${ }^{1,2}$, Maarten A. Hogervorst ${ }^{1}$, Ysbrand van der Werf ${ }^{3}$

41 Perceptual and Cognitive Systems, TNO, Soesterberg, The Netherlands

52 Human Media Interaction, University of Twente, Enschede, The Netherlands

63 Anatomy and Neurosciences, VU University Medical Center, Amsterdam, The Netherlands

7 Corresponding author:

8 Jan van Erp

$9 \quad$ TNO Perceptual and Cognitive Systems

10 Kampweg 5

11 3769DE Soesterberg

12 The Netherlands

$13+31(0) 888665982$

14 Jan.vanerp@tno.nl 


\section{Toward physiological indices of emotional state driving future ebook \\ 17 interactivity}

Ebooks of the future may respond to the emotional experience of the reader. (Neuro-) physiological measures could capture a reader's emotional state and use this to enhance the reading experience by adding matching sounds or to change the storyline therewith creating a hybrid art form in between literature and gaming. We describe the theoretical foundation of the emotional and creative brain and review the neurophysiological indices that can be used to drive future ebook interactivity in a real life situation. As a case study, we report the neurophysiological measurements of a bestselling author during nine days of writing which can potentially be used later to compare them to those of the readers. In designated calibration blocks, the artist wrote emotional paragraphs for emotional (IAPS) pictures. Analyses showed that we can reliably distinguish writing blocks from resting but we found no reliable differences related to the emotional content of the writing. The study shows that measurements of EEG, heart rate (variability), skin conductance, facial expression and subjective ratings can be done over several hours a day and for several days in a row. In follow-up phases, we will measure 300 readers with a similar setup.

Keywords: reading, creativity, emotion, neurophysiology, brain-computer interfaces, ebook, interactivity, EEG, multimedia, Human-Computer Interaction.

\section{Introduction}

The sales of ebooks are rapidly increasing and are expected to surpass that of printed books in the near future. In its basic form, an ebook is an electronic version of the printed book. However, the devices used to access an ebook (ereader, tablet, etc.) have more capabilities than just displaying the book and turning pages on request of the reader. The device may enable true bidirectional interaction with the reader, which is a significant innovation compared to the onedirectional printed book. This interactivity may substantially change the future of the ebook as artistic form and may result in new interactive media products that only slightly resemble the basic version of the printed book as sold today. 
potential of interactive ebooks. One of the key questions is which reader parameter or actions

(other than turning pages) are useful for interactive ebooks. One of the driving forces behind this

exploration was the prominent Dutch writer Arnon Grunberg who also had a genuine interest in

what his readers actually experience while reading his work, or more generally stated: “Is

reading a novel good for you?" (the writer himself takes a devil's advocate stance and postulates

the possibility that reading literature has a detrimental influence [1]). From neuroscientific data,

we know that reading is a complex task involving many brain areas ([2], see [3]for a recent

review and [4] for individual differences in narrative comprehension) and that reading can (at

least temporarily) alter connectivity in an individual's brain $[5,6]$. However, just reading text

doesn't make one more social or empathetic. This may only happen after so-called "emotional

transportation" $[7,8]$, i.e. as a reader one needs to be involved at an emotional level. It is

postulated that there are no effects of reading non-fiction and also no effects of reading fiction

when there is no emotional transportation [9]. A similar concept (immersion) is used in the

"fiction feeling hypothesis" [10] which postulates that negative, high arousal text activates the

affective empathic network [11] which facilitates immersion in the text. In an experiment,

participants read neutral and fearful sections of the Harry Potter saga and the results indeed

showed a relation between neuronal activation pattern and subjectively rated immersion.

61 Emotional experience is not only an essential catalyst, but also important in choosing which

62 book to read, experiencing the content [12] and interpreting the narrative [13].

All the above led us to develop a research project to measure readers' emotions while 
66 technology. In addition, we were interested in measuring the emotions of the writer during the

67 writing process to be able to compare the reader's emotional state while reading a certain

68 paragraph to that of the author during writing that same paragraph. Capturing the emotional state

69 of the writer (both through neurophysiology and subjective ratings) became our case study and is

70 reported in this paper to illustrate the use of sensor technology and to investigate whether

71 prolonged physiological measurements are feasible in a real life situation. The framework

72 described here is the basis for follow-up studies in which several hundreds of readers will read

73 the book before publication while being measured with a similar setup as used here with the

74 author [14]. The applied, real life perspective guided the selection of theoretical models and

75 measurement methods.

\subsection{Art, beauty and neuroscience}

There is a growing interest in using neurophysiological measures to assess media, including paintings, music and films. Research in this area is still at the forefront of cognitive neuroscience and results and theoretical foundations are still under debate. An important question that has fascinated and divided researchers from both the neurosciences and the humanities is whether brain activity can provide insight in what true art and beauty is. From an applied point of view, the relevant question is whether an individual's brain pattern is informative of his or her appraisal of the piece of art. Research of Zeki and colleagues, amongst others, has shown that there is a functional specialization for perceptual versus aesthetic judgments in the brain [15] and that there is a difference in activation pattern for paintings experienced as beautiful by an individual and those experienced as ugly. This finding is independent of the kind of painting: portrait, landscape, still life, or abstract [16]. Hasson and colleagues [17] used fMRI to assess the effects of different styles of filmmaking on brain 
89 patterns and suggest that neurophysiological sensing techniques can be used by the film industry

90 to better assess its products. The latter was done by [18] who measured skin conductance as an

91 affective benchmark for movies and by [19] who measured cardiovascular and electrodermal

92 signals and found a high degree of simultaneity between viewers, but also large individual

93 differences with respect to effect size. So far, interactivity based on viewers emotional state has

94 not moved beyond a few artistic experiments: "unsound" by Filmtrip and SARC

95 (http://www.filmtrip.tv/) and "Many Worlds" by Alexis Kirke (http://www.alexiskirke.com/).

96 In this paper we look at the (applied) neuroscience behind both the creative and the

97 emotional brain and how emotional state can be captured using wearable, mobile technology that

98 is usable while reading an ebook. We will also explore the possibilities opened up after capturing

99 a reader's emotional state and what the ebook of the future might look like. The paper also

100 presents the data of the writer during the creation of emotional text [20].

\section{The emotional brain}

102 Stimuli evoking emotions are processed in the brain through specific pathways and with the

103 involvement of several brain areas. In other words, the emotional brain is a network of

104 collaborating brain areas and not a single location [21,22]. The majority of the sensory

105 information entering the brain goes to the primary sensory areas, but a small part of the

106 information goes to the amygdala, part of the limbic system deep inside the human brain. A main

107 driver of the amygdala is danger: in case of a potential threat to the organism, the amygdala is

108 able to respond quickly and prepare the body for action without much stimulus processing. The

109 amygdala enables the release of stress hormones leading to peripheral effects, for instance

110 increased heart rate to pump more blood to the lungs and muscles. After the amygdala,

111 processing continues through the cingulate cortex, the ventromedial prefrontal cortex and finally 
112 the dorsolateral prefrontal cortex. Only in the dorsolateral prefrontal cortex is the processing

113 stream through the amygdala integrated with the more cognitive processing stream from the

114 sensory cortices. The emotional experience is a result of the interpretation of both processing

115 routes taking into account the context and previous experiences. This integration and

116 interpretation of information is a typical function of the prefrontal cortex [23].

\section{2.1 Psychological framework of emotions}

118 Before we can discuss how we can measure emotional state, we should first look into the

119 frameworks to classify emotions. There are many psychological frameworks available. Classic

120 work by Paul Ekman [24] and James Russell [25] shows that there are several basic emotions:

121 fear, disgust, anger, happiness, sadness and surprise. This set of six basic emotions has been

122 expanded through the years with numerous subclasses. From a neuroscientific point of view, an

123 important question is whether these emotions each have their own (unique) neuronal location or

124 circuit (i.e. a discrete model [26]), or vary along several independent dimensions (i.e, a

125 dimensional model [27]), a matter that is still under debate. As described above, experiencing an

126 emotion is the result of the integration and interpretation of numerous information streams by an

127 extended network of brain areas which makes a discrete model unlikely. Therefore, we adopt a

128 dimensional model, or more specifically the circumplex model of emotion [25,28,29] in which

129 emotions are plotted in two dimensions: arousal and valence. For instance, anger is linked to

130 negative valence and high arousal, sadness to negative valence and low arousal, and happiness to

131 positive valence and high arousal and contentment to positive valence and low arousal. This

132 model is commonly used to investigate for instance emotional words, facial expressions, and

133 affective states. 
134 The circumplex model of emotion stems from the ratings of individual, written words.

135 Neuro imaging studies confirm the two-dimensional model of valence and arousal and although

136 there may be complex interactions between both dimensions, they both have a different signature

137 of brain activation, spatially as well as temporally. Arousing words show a different pattern

138 (compared to neutral words) mainly in the early processing stages (i.e. within $400 \mathrm{~ms}$ after

139 presentation including the following ERP components: early posterior negativity (EPN), P1, N1,

140 P2, and N400) while the difference between positively versus negatively valenced words shows

141 in later processing stages (between 500 and $800 \mathrm{~ms}$ after presentation including the late positive

142 complex (LPC)) [30]. In the spatial domain, arousal is linked to amygdala activity and valence to

143 the cingulate cortex and the orbitofrontal cortex [31-35]. Excellent reviews are given by [36] and

144 [37]. Based on her review, Citron [38] comes to the conclusion that positive and negative

145 valence may differ with respect to the cognitive functions they activate and are not necessarily a

146 continuous dimension. Although a novel is more than a collection of individual words, there has

147 been very little research on the physiological reactions to reading larger pieces of text (see the

148 first section of this paper), but a lot to reading individual words. This project aims to fill that

149 void.

\subsection{Emotion classification using neurophysiological measures}

151 With the circumplex model as point of departure, we can start to identify physiological signals

152 that reflect the arousal and valence of emotions and that can potentially be measured while

153 reading outside a laboratory environment. We will look at a broader range of methods used to

154 induce an emotional state than written words and at a broader set of physiological measures than

155 EEG and fMRI. For example, [38] induced emotional state by letting subjects imagine pleasant,

156 unpleasant, aroused and relaxed situations and measured effects on EEG, heartrate, skin 
157 conductance, skin temperature and respiration.

158 2.2.1 Valence

159 Valence requires central nervous system indices as it is less clearly reflected in peripheral

160 measures. Wearable sensors like EEG are not able to measure activity in deeper structures like

161 the limbic system but as reviews show [36,37], valence is strongly linked to later processing

162 stages involving more superficial brain structures related to cognitive processing.

163 Valence is reflected in (late) ERP components [39-41], in the power in specific EEG

164 frequency bands like alpha [42,43], in the relative power in different EEG bands [44] and in

165 asymmetrical alpha activity in the prefrontal cortex [23,45-47] indicating increased left

166 prefrontal cortex activity for positive valence and increased right prefrontal cortex for negative

167 valence. However, power in the different frequency bands and hemispheric asymmetry are under

168 the influence of many factors, which may only partially correspond to emotional valence. For

169 example, hemispheric asymmetry has been linked to stress [48] and the tendency to approach

170 versus to avoid stimuli [49], and low power in the alpha band may be caused by the fact that

171 stimuli with high valence may attract more attention [50,51].

\section{2.2.2 Arousal}

173 Arousal is less clearly linked to brain activation patterns except for activity in the amygdala and

174 the reticular formation [29], which are difficult to measure with wearable sensors like EEG.

175 However, arousal is reasonably clearly reflected through a relatively strong activation of the

176 sympathetic as compared to the parasympathetic autonomous nervous system. Arousal can be

177 measured peripherally through, for instance, skin conductance (increasing conductance with

178 increasing arousal [52]), heart rate variability (HRV), especially high frequency HRV as this is 
179 exclusively affected by the parasympathic system (reduced high frequency HRV with increased

180 arousal [53]), pupil size, heart rate (HR) and respiration frequency (all increased with increased

181 arousal, although this pattern is not consistent over studies, see [54] for an elaborate overview).

\section{$182 \quad 2.3$ Current state of the art in (applied) emotion capture}

183 The state-of-the-art in emotion detection using neurophysiological indices is that we are able to

184 distinguish several valence and arousal levels in a lab environment when subjects are sitting still

185 and sufficient control data is gathered beforehand to train classification algorithms (see [55] for

186 an overview). However, it is important to note that the relation between physiology and emotion

187 is not straightforward. Different studies with different stimuli and contexts report different types

188 of correlations [54,56]. It is thus important to study relations between (neuro-) physiology and

189 emotion within the context and under the circumstances of interest [57,58].

190 An important step in this project, is to bring neurophysiological signals out of the lab and

191 explore their potential value in daily life [55,58]. Monitoring and using the (neuro-)

192 physiological signals of readers is new, and entertainment in general is a good first case to

193 transfer the technology from the laboratory to real life. This transition will come with several

194 challenges ranging from coping with external noise due to movement artifacts, multitasking

195 users, and usability aspects such as prolonged usage [58-60]. First steps in this transition have

196 recently been made in studies investigating EEG signals in gaming [61] and into music

197 perception in realistically moving participants [62]. Here we also present the case of the writer

198 wearing physiological sensors for several hours a day and for 9 days in a row.

\section{The creative brain}

200 The current case study focused on the writer and his emotional signals during the creative 
201 writing process. Our primary goal was to implement and learn about the transition from

202 laboratory to real life before upscaling the set-up to hundreds of readers, and to capture the

203 emotional signals of the writer as function of the emotional content of the written paragraphs.

204 We deemed it worthwhile, nevertheless, to have a quick look at the creative brain as well. Most

205 people would agree that creative abilities make us unique in the animal kingdom. Interestingly,

206 we understand little of the processes that drive or facilitate creativity and still debate on the

207 definition of creativity, although most agree upon the importance of both novelty and usefulness

208 (see [63] for an elaborate discussion).

209 Similar to the neuroscience of art and beauty, neuroscientific research into creativity can

210 still be characterised as embryonic and neuroscientific models are not widely established yet.

211 Like emotion, creativity is not related to a single brain area but rather to networks of brain areas.

212 Based on an extensive review, Dietrich and Kanso even state that "creativity is everywhere" [64]

213 see also [65]. Having said that, recent neuroimaging studies seem to show that creativity involves

214 common cognitive and emotional brain networks also active in everyday tasks, especially those

215 involved in combining and integrating information. For the current project, it is useful to

216 distinguish two different types of creative processes as described by Dietrich [66]. The first can

217 be called controlled creativity often in relation to finding creative solutions for a particular, given

218 problem. This creative process is controlled through the prefrontal cortex [67] that guides the

219 search for information and the combination of information within a given solution space. A

220 powerful mechanism which is bound, though, by limitations of the prefrontal cortex, for instance

221 with respect to the number of solutions that can be processed in working memory. The second

222 type can be named spontaneous creativity, often in relation to artistic expression. This form of

223 creativity comes without the restrictive control from the prefrontal cortex, and the process differs 
224 from controlled creativity qualitatively (e.g. solutions are not bound by rational rules like the

225 rules of physics) and quantitatively (the number of solutions is not restricted by for instance the

226 limited capacity of working memory). Spontaneous creativity is linked to unconscious processes

227 (of which dreaming may be an extreme form). However, the prefrontal cortex becomes involved

228 in spontaneous creativity when solutions will eventually reach the conscious mind, and the

229 prefrontal cortex is required to evaluate them and bring them to further maturity.

230 Recent data show us that less activity in the dorsolateral prefrontal cortex links to

231 increased spontaneous creativity in, for instance, musicians [68,69], and increased activation to

232 increased controlled creativity. Results also show that there is a burst of wide-spread gamma

233 activity about $300 \mathrm{~ms}$ before the moment of insight in spontaneous creativity. Gamma activity is,

234 amongst other features, linked to binding pieces of information. A burst of gamma activity is

235 indicative of finding (and binding) a new combination of chunks of (existing) information. Fink

236 and Benedek [70] underline the importance of internally oriented attention during creative

237 ideation in a more general sense, reflected in an increase in alpha power.

238 Creativity is also linked to hemispheric asymmetry. A meta-analysis [71] showed that the

239 right hemisphere has a larger role in creative processes than the left hemisphere. This is

240 confirmed by patient research [72,73]. A lesion in the right medial prefrontal cortex hinders the

241 creation of original solutions while a lesion in the left medial prefrontal cortex seems to be

242 beneficial for spontaneous creativity. However, experiments with creative students [74] and

243 extremely creative professionals from science and arts [75] both show bilateral cerebellum

244 involvement, seemingly confirming the statement that "creativity is everywhere in the brain".

245 However, these findings are general findings and may not be applicable to the creative

246 writing process [76]. For instance, creative writing seems to result in increased activity in the left 
247 prefrontal cortex (presumably because of its links to important language areas in the left

248 hemisphere), except when writing emotional text, for which activity in the right hemisphere

249 seems to be greater. This shows that the body of knowledge on the creative brain is growing but

250 still limited and identifying neural correlates of the creative writing process requires further

251 research. Another interesting debate is whether creative writing is a skill one can develop like

252 skilled behavior in sports and music, or possibly even non-creative, non-fiction writing like

253 scientists and journalists do. Lotze and colleagues found that the caudate nucleus (involved in

254 skilled behavior) was active in experienced creative writers but not in novices [77,78], indicating

255 that creative writing can indeed be a (trainable) skill.

\section{The case study}

257 4.1 Methods

\section{4.1.1 Participant}

259 Arnon Grunberg (http://www.arnongrunberg.com/) participated in the study. Arnon Grunberg

260 was born in 1971 and has lived in New York since 1995. He writes novels, short stories,

261 columns, essays and plays. His work was awarded with several national and international prizes

262 and translated into 30 languages. He participated voluntarily, being aware that his participation

263 would not be anonymous. All data were collected in November 2014 in Arnon's apartment in

264 New York. The Institutional Review Board of TNO Human Factors (TCPE Soesterberg, The

265 Netherlands) approved the study after inclusion of specific sections in the informed consent

266 regarding privacy and data dissemination. Arnon read and signed the informed consent before

267 data gathering began. 


\subsubsection{Apparatus}

269 We used commercially available hardware and software to record physiological parameters,

270 facial expression and text entry. In addition, paper and pencil were used for subjective

271 questionnaires described in the next section.

272 All neurophysiological signals were recorded using a wearable Mobita® 32-channel

273 physiologic signal amplifier system sampling at 1000Hz (TMSi, Hengelo, The Netherlands,

274 http://www.tmsi.com/). The available channels were used for EEG (28 TMSi water based

275 electrodes, see Figure 1 for the layout of the electrodes), ECG (two pre-gelled disposable TMSi

276 snap electrodes) and Endosomatic Skin Potential ESK (pair of TMSi finger electrodes). The

277 Mobita ${ }^{\circledR}$ has built-in accelerometers which we used to $\log$ possible activity of the writer and to

278 synchronize the physiological data to other data gathered through a Noldus Observer XT ${ }^{\circledR}$

279 system (Noldus IT, Wageningen, The Netherlands, http://www.noldus.com/). This system

280 recorded the images from two IP cameras (one providing an overview of the work space and one

281 providing a close-up of the writer's face for later analysis of his facial expression), a continuous

282 screen dump of the writer's PC screen, and the writer's keystrokes (Noldus uLog tool ${ }^{\circledR}$ ). The

283 writer used his normal work space and own PC, see Figure 2. 

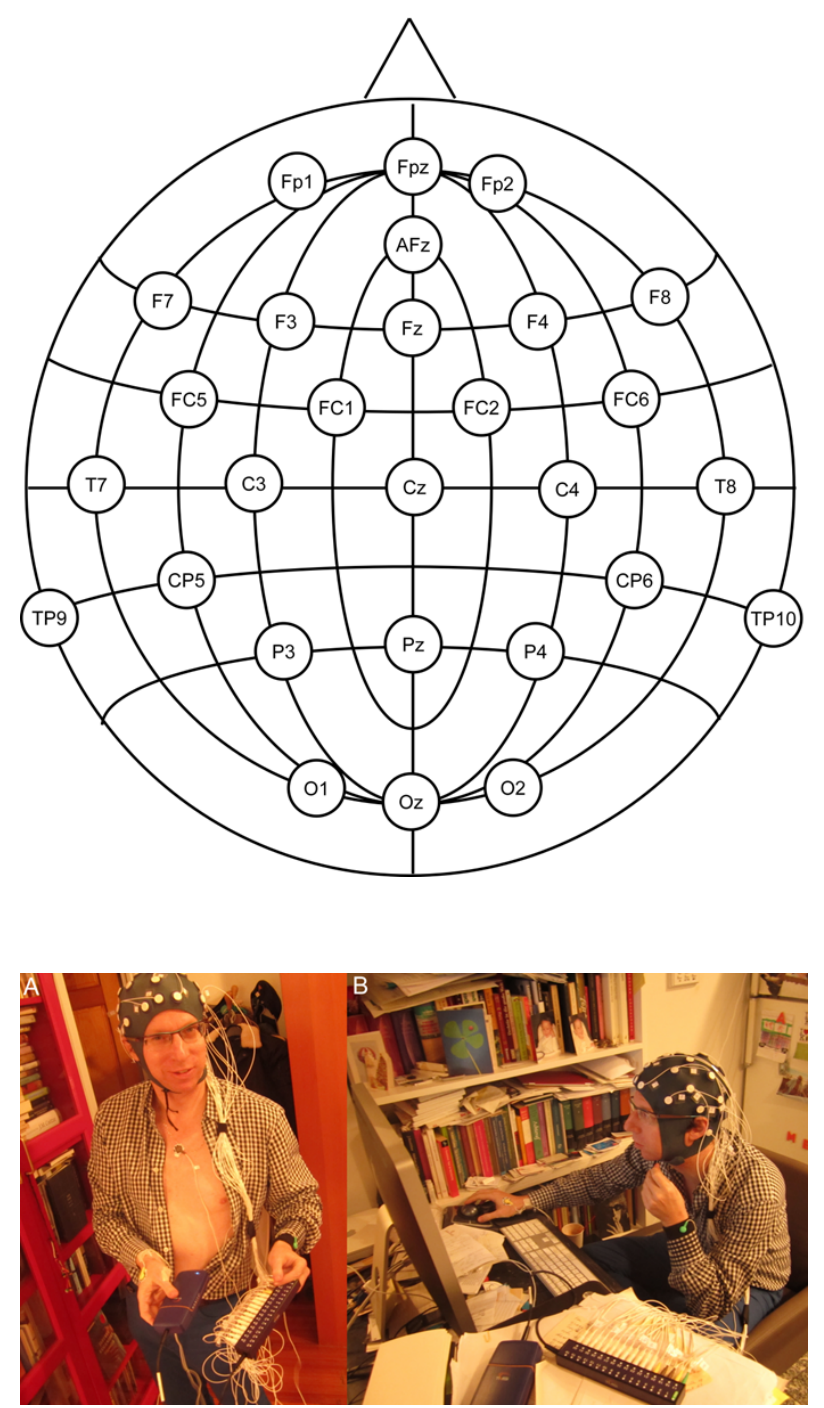

290 Table 1 gives the outline of the experimental protocol for one day. Table 2 gives the details on 291 the experimental protocol. 


\subsubsection{Data processing}

295 Our intention was to use the calibration sessions of each day to identify differences in

296 physiological markers that could be linked to the emotional content of the written paragraph.

297 When we can reliably establish this 'ground truth', it could consecutively be used to analyze the

298 data gathered during the writing blocks. After checking the synchronization between the

299 different data streams, the physiological data of the calibration session were separated in 10

300 epochs corresponding to $1 \mathrm{~min}$ rest eyes open, 1 min rest eyes closed, $6 \times 2$ min 'emotional

301 writing' (each corresponding to one of six different emotional pictures and descriptors), and

302 again $1 \mathrm{~min}$ rest eyes open, 1 min rest eyes closed.

$303 E E G$. The EEG data were processed using the following pipeline: re-referencing to channel

$304 \mathrm{TP} 10$, rejection of channels with very large variance (channels $\mathrm{O} 1, \mathrm{Oz}$ and $\mathrm{O} 2$ were very noisy

305 and removed completely from the dataset), band pass filtering 0.5 to $43 \mathrm{~Hz}$, and down sampling

306 to $250 \mathrm{~Hz}$. Initialy, the EEG data of the remaining channels were used in an Independent

307 Component Analysis (ICA) to identify and remove potential artifacts. However, the ICA

308 revealed that potential artifacts were non-stationary (i.e. changing over time) and therefore

309 difficult to identify and thus no more data were removed. The power in different frequency

310 bands: delta $(0-4 \mathrm{~Hz})$, theta $(4-8 \mathrm{~Hz})$, alpha $(8-13 \mathrm{~Hz})$, SMR $(13-16 \mathrm{~Hz})$, Beta $(16-30 \mathrm{~Hz})$ and

311 gamma $(30-80 \mathrm{~Hz})$ were used as features in the classification.

312 Peripheral physiology. As a measure of heart rate, we determined the mean interval between

313 successive R-peaks in the ECG (RRI) for each epoch and converted this to mean Heart Rate 
$314($ meanHR $=1 /$ meanRRI). Four measures of heart rate variability were derived. The root mean

315 squared successive difference between the RRIs (rmssdRRI) reflects high frequency heart rate

316 variability. We also determined heartrate variability in the low, medium and high band using a

317 spectral analysis (HRVlow, HRVmed, HRVhigh). High-frequency heart rate variability was

318 computed as the power in the high frequency range $(0.15-0.5 \mathrm{~Hz})$ of the RRI over time using

319 Welch's method applied after spline interpolation; similarly for mid-frequency $(0.07-0.15 \mathrm{~Hz})$

320 and low-frequency $(0-0.07 \mathrm{~Hz})$ heart rate variability. No anomalies were present in the ECG data

321 so no data was removed. From the ESK, the mean ESK over the epochs was calculated. For the

322 ESK we removed one outlier (contentment epoch on day 2).

323 Classification analysis using EEG and peripheral physiology features. To determine how well

324 various feature sets could predict the emotional state of the author during the calibration session

325 we performed a classification analysis. Classification was performed using the Donders Machine

326 Learning Toolbox [79]. Two types of classifiers were used: a linear Support Vector Machine

327 (SVM) and an elastic net model with logistic regression [80]. As input we used the features that

328 were standardized to have mean 0 and standard deviation 1 on the basis of data from the training

329 set. One-tailed binomial tests were used to determine whether classification accuracy was

330 significantly higher than chance.

331 Facial expression. The images from the close-up camera were analysed offline using Noldus

332 FaceReader software. Output for each epoch are intensity values for the following

333 classifications: Neutral, Happy, Sad, Angry, Surprised, Scared, Disgusted.

334 Subjective questionnaires. The data of the feelings grid, VAS, and DES full questionnaires was

335 not pre-processed but directly analysed. We only statistically analysed the main effects of day (9

336 levels) and session (start of day and end of day for DES full, and start of day, end of block 1, end 
337 of block 2, end of day for feelings grid and VAS). The DES full scores were analysed using non-

338 parametric statistics with alpha level Bonferroni adjusted for the number of comparisons.

339 Feelings grid and VAS scores were analysed with a parametric ANOVA.

\subsubsection{Procedures}

341 We started the measurements on the day the writer started with a new novella to be used in phase

3422 of the project. We adjusted the measurements to his usual daily writing schedule comprising

343 two blocks: one in the morning and one in the late afternoon or early evening. He normally

344 writes for about two hours and fills the time in between with other activities (including other

345 writing activities). During a writing block, he was engaged in other activities as well like

346 answering emails and phone calls etc., but never during the instrumentation and calibration. All

347 activities during the measurement blocks were logged by the experimenter who was always

348 present during the measurements. We measured for nine consecutive days. At the end of the day,

349 the experimenter and the writer would make a specific schedule for the next day. The writer also

350 reflected on his experiences over the day, including the user experience of wearing the

351 equipment and being observed. The day before the start of the experiment, the protocol,

352 instructions etc. were explained in great detail, the writer signed the informed consent, his

353 workplace was instrumented and the equipment tested. Besides the addition of the equipment,

354 the writer's workplace was not altered in any way to give the writer the best opportunity to

355 behave as usual. On each measurement day, the experimenter came to the apartment as

356 scheduled and followed the protocol as detailed above. At the end of the day, all data were

357 encrypted and saved to an external hard disk. 


\subsection{Results}

359

360

361

362

363

364

365

366

367

368

369

370

371

372

373

374

375

376

377

378

379

\subsubsection{Classification of baseline vs. emotion conditions in the calibration blocks}

First, we determined whether the feature set contained information to discriminate the baseline conditions (Eyes Open and Eyes Closed) from the emotional (writing) epochs using binary classification (baseline vs. non-baseline). For this purpose we performed a 'leave-one-day-out' cross validation using the SVM classifier. This method is to be preferred over random $\mathrm{N}$-fold cross validation since it better accounts for possible correlations between data during the day [81]. Still, the results when using random folds were found to be comparable to the results of the analysis presented here. It is also important to compensate for the imbalance in the number of conditions, with 36 baseline blocks and 54 emotional blocks in the set. All reported performance scores follow a binomial distribution and the variability of the binomial distribution follows directly from the average score and the number of measurements (the distribution is not well approximated with a Gaussian distribution and therefore the variance is not a good indicator of the variability in the results). For larger number of measurements the variability is approximately equal to $p^{*}(1-p) / N$ (with $p$ estimated by the score, $N$ the number of measurements).

When all six physiology features (i.e. meanHR, HRVlow, HRVmed, HRVhigh, rmssdRRI, meanESK were used as input to the classifier the average model performance (over all days) was $71 \%$, with a hit-rate (score for correctly classifying baseline blocks) of $58 \%$ and a False-Alarm-rate (FA-rate, i.e. fraction of falsely classified emotional blocks) of 20\%, resulting in an equal cases (in the situation in which both conditions occur equally frequent) performance of $69 \%(\mathrm{p}<.01)$. Individual ANOVAs with condition as independent variable (baseline vs writing) and physiological measure as dependent variable showed significant differences for the 
380 heart rate variability measures only (all $F$ values $>5.83$, all $p$ values $<.02$ ). Figure 3 gives the 381 HR and rmssd RRI as function of calibration block.

382
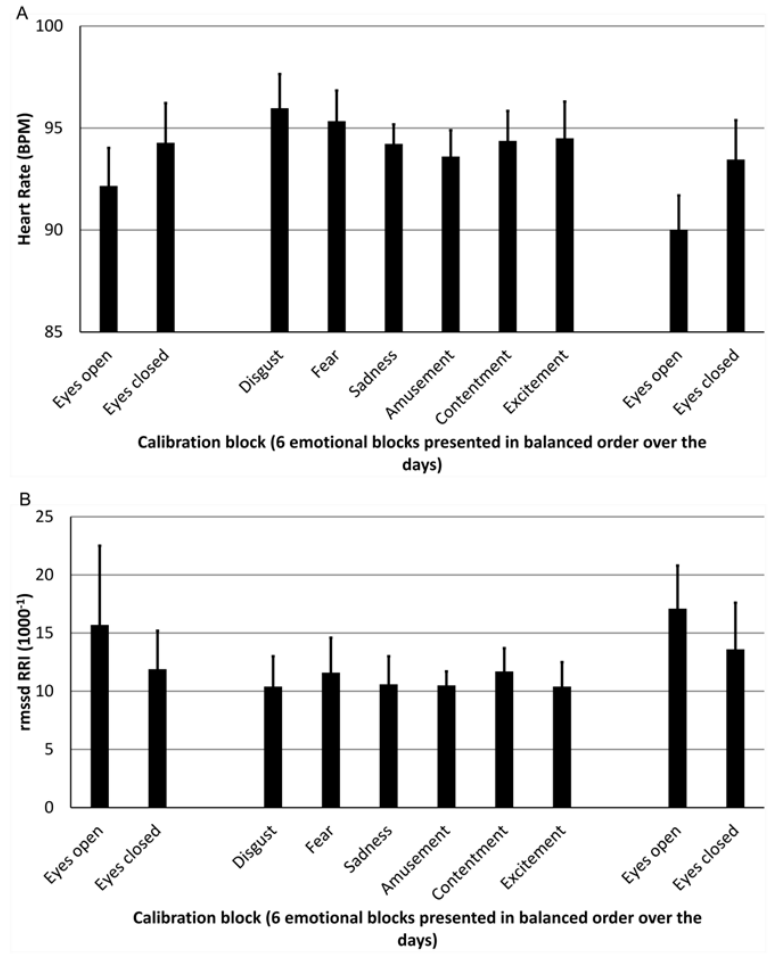

$383<<$ Figure 3. Results of the physiological measures Heart Rate (A) and rmssd RRI (B) as

384 function of the different calibration blocks. Eyes open and Eyes closed blocks were measured

385 before and after the emotional blocks. The order of the emotional blocks was balanced over days.

Error bars denote the standard error of the mean. $>>$ 

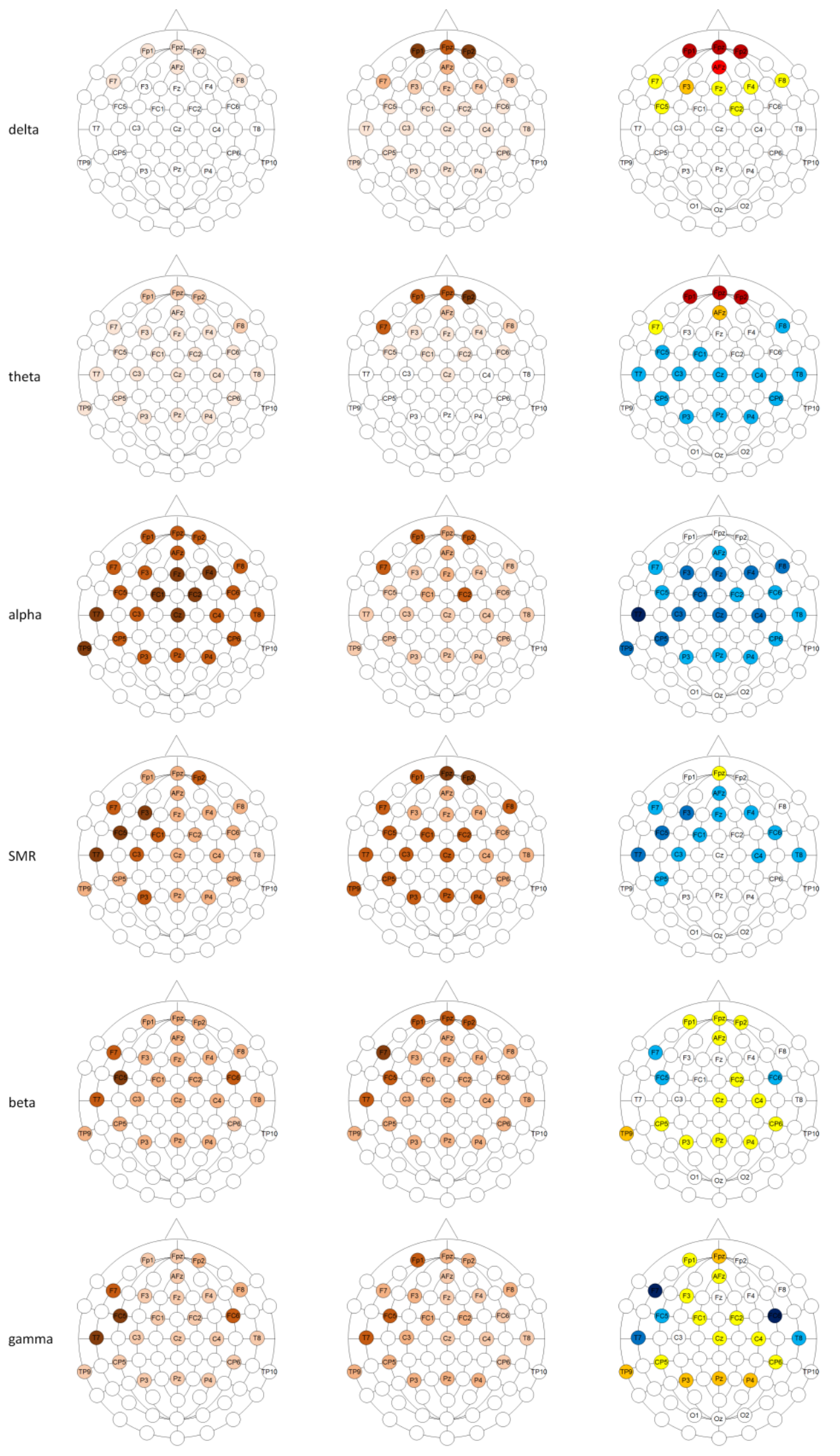

$.15<$ weight

$.10<$ weight $<.15$

$.05<$ weight $<.10$

$-.05<$ weight $<.05$

$-.10<$ weight $<-.05$

$-.15<$ weight $<-.10$ 
$<<$ Figure 4. The left column shows the power in the different frequency bands in the rest

blocks (eyes open and eyes closed combined) and the middle column in the writing blocks. The right column gives the weights of the features in the classification model.>>

391

392

Figure 4 summarizes the power distribution for the different frequency bands averaged

393

over the rest epochs (left column) and the writing epochs (middle column). When the EEG

394

features were used as input, the average model performance (over all days) was $92 \%$ with a hit-

rate of $86 \%$ and a False-Alarm-rate (FA-rate) of $4 \%$, resulting in an equal cases performance of

$91 \%(\mathrm{p}<.01)$. The model weights are depicted in the right column of Figure 4 . Inspecting Figure

4 shows that there are three major differences between rest and writing reflected in the model

weights. The writing blocks show: (1) increased frontal power in the delta and theta bands, (2)

wide spread suppression of alpha, and (3) central increase in beta and gamma activity. The first

effect is most likely caused by eye movements. The second effect relates to the suppression of

401

the brain's idle state during rest. The increase in gamma activity may be related to creative

processes as described in Section 3, but gamma components are also susceptible to muscle

artifacts caused by e.g. jaw clenching and forehead movements. If we only use the alpha and movements. performance was also 92\%, with a hit-rate of $89 \%$ and a False-Alarm-rate (FA-rate) of 6\%, 
410 to using only EEG features, indicating that the added value of incorporating features other than

411 EEG ones is small in this case.

412 A closer inspection of the feature weights in the classification model showed that the

413 highest weights are attributed to the delta $(0-4 \mathrm{~Hz})$ and theta (4-8) bands in channels Fp1, Fpz,

414 Fp2 (i.e. frontal channels). The equal class performance of a classification model using only

415 these six features is 0.84 (compared to 0.92 for a model using all features). Slow (0-4 Hz)

416 frequency bands of EEG may pick up eye movements and should be evaluated with caution

417 (please note that eye movements were not removed from the EEG data). Indirect measurement of

418 eye movements in the EEG signal masks the information in the primary EEG. Even in case it is a

419 reliable classifier for the current experimental setup, we consider it an artifact.

\subsubsection{Classification of valence and arousal in the calibration blocks}

421 Model performance was determined for classifying low vs. high valence and arousal using 10-

422 fold cross validation using a range of parameters:

423

424

425

426

427

428

429

430

431
- Features from EEG, physiology or both,

- Binary classification for predicting outcomes higher than the median value or using only the extreme values, i.e. lower than the 0.33 -quantile or higher than the 0.66 -quantile,

- Using raw or normalized features, in which case the features were normalized by dividing by the average feature value for the Eyes-Open conditions (for that day),

- Using SVM or elastic net classifier with logistic regression.

In none of the cases did we find classification performance deviating significantly from chance performance. Since classification performance using the whole set of EEG data did not result in above-chance performance, we did not continue using specific subsets only, e.g. to look at the 
432 power in specific EEG frequency bands like alpha $[42,43]$, at the relative power in different EEG

433 bands [40] and at asymmetrical alpha activity in the prefrontal cortex. Individual ANOVAs on

434 the physiological measures confirmed these observations: all $F$-values $<0.63$ and all $p$ values $>$

435.67 , see also Figure 3. Because building a reliable valence and arousal classification algorithm

436 using the calibration data turned out to be impossible, we could not further classify the novel

437 writing data.

438

439

440

441

442

444 445

446

\subsubsection{Facial expression in the calibration blocks}

We used the FaceReader ${ }^{\circledR}$ output directly in the analysis and found no significant differences between the different emotional paragraphs. Generally, the facial expression of the writer was classified as neutral (about 30\%), sad (about 25\%) or angry (about 20\%). The remaining 25\% was dispersed over happy, surprised, scared and disgusted.

\subsubsection{Subjective questionnaires}

The DES full showed neither differences over the days (1-9) nor over sessions (start - end of day). Analysis of the feelings grid scores showed a significant effect of arousal over sessions: $F(3,31)=4.57, p<.01$. A post-hoc LSD test showed a significant difference between start of the day and the end of block 2 and end of the day. The analyses of the VAS scores showed no effect over days, but a large effect over sessions of happy: $F(3,31)=3.65, p<.03$, optimistic: $F(3,31)$ $=6.28, \mathrm{p}<.01$ and flow: $\mathrm{F}(3,31)=6.76, \mathrm{p}<.001$, and a trend for relaxed: $\mathrm{F}(3,31)=2.38, \mathrm{p}<$ .09. The means of the significant effects over session are presented in Figure 5. The figure shows that happy, optimism and flow are rated high at the start of the day but systematically decrease over the writing sessions with a stabilization or reversal at the end of the day. For arousal, this effect is inverted. These trends are confirmed by post-hoc LSD tests. 
was uncomfortable at the start but that he got used to wearing the cap and the other physiological

456

457

458

459

460

461

462

463

464

465

466

467

468

469

470

471

sensors. He experienced the cameras as more obtrusive and disturbing than the physiological

sensors. He elaborated on this in several public interviews (e.g., in The New York Times:

www.nyti.ms/1dGxkFR).

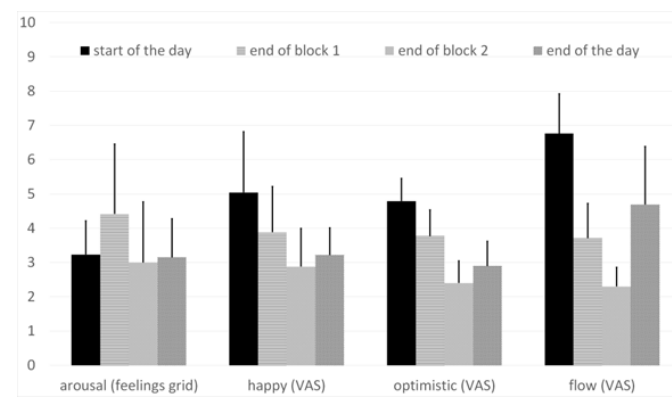

$<<$ Figure 5. Significant changes in subjective ratings over the course of a writing day. $>>$

\subsection{Discussion}

\subsubsection{Set-up and user experience}

The case study primarily focussed on measuring neurophysiological indices over prolonged periods outside a laboratory environment before applying the technology in a large scale experiment with the readers of the novel. Inspection of the signals revealed that, except for EEG channels $\mathrm{O} 1, \mathrm{Oz}$ and $\mathrm{O} 2$, we were able to record reliable signals in a real life situation using wearable / wireless sensor technology and that the setup was comfortable enough for the writer to work for hours a day wearing the sensors. The noise in the occipital channels may be caused by (neck) muscle activity related to mouse and keyboard actions. The ICA analysis indicated that potential artifacts were non-stationary (i.e. changed over time), an effect similar to 
472 what we find with readers [14]. Non-stationarities may be more common in real-world,

473 multitasking environments and hamper identification and removal of artifacts [58]. This

474 increases the relevance of including EMG and EOG sensors to the sensor suite. Data analysis

475 may also benefit from a higher electrode density allowing to apply more advanced techniques for

476 artifact removal and EEG analysis. Recent electrode developments may enable this without

477 reducing usability and comfort over prolonged periods of use.

478 Although measuring physiology outside a well-controlled laboratory environment is

479 challenging, the data show reliable differences between resting state and writing, which indicates

480 a sufficient signal-to-noise ratio in the data. It could still be the case that this "writing detector" is

481 triggered by artifacts like eye movements or muscle activity that comes with typing (however,

482 the EEG channels most prone to these muscle artifacts $(\mathrm{O} 1, \mathrm{Oz}$ and $\mathrm{O} 2)$ were removed from the

483 dataset). If we look at the weight of the different features in the classification algorithm, we see

484 that most weight is attributed to the delta $(0-4 \mathrm{~Hz})$ and theta (4-8) bands in the frontal channels

485 (Fp1, Fpz, Fp2). Low frequency bands should be evaluated with caution as they may reflects eye

486 movements rather than signals in the primary EEG. This is especially relevant for the current

487 dataset since (eye movement) artifacts were non-stationary and could not be reliably identified

488 and removed. However, the classifier is also based on suppression of alpha and increased central

489 gamma activity during writing. This matches with the expected pattern for creative writing

490 although one should note that gamma can also be affected by e.g. jaw clenching artifacts. In

491 addition, the current differences in physiology like increased heartrate and decreased heartrate

492 variability (see Figure 3) do fit with an interpretation of low (rest) and high cognitive activity

493 (writing) and not just with simple muscle activity. To exclude the aforementioned artifacts, a 
494 comparison should be made between writing about an emotion, writing down mundane

495 instructions, and for instance copying text or making random typing movements.

496

498

499

500

501

502

503

504

505

506

507

508

509

510

511

512

513

514

515

516

\subsubsection{Neurophysiology of emotional writing}

Since there are no data available about neurophysiological correlates of emotional

writing, we based our expectations on research into physiological responses based on presenting many repetitions of, for instance, emotional pictures or sounds. In this domain, recent

experiments show that changes in emotional state can also be reliably identified with a restricted number of repetitions or even single trial (especially for longer epochs like in our study).

Therefore, we expected to be able to see changes in physiological state as a function of emotional content, despite the limited number of repetitions. However, we were not able to link specific neurophysiological indices to the emotional content of the writing. We have three possible explanations: (1) the quantity and/or quality of the data was not sufficient, (2) writing is a cognitive rather than an emotional task for this particular author, and (3) the task involved a multitude of emotional, creative and cognitive processes concealing the single-task indices found in single-task laboratory experiments. The first explanation pleads for expanding the data set using more authors and possibly more sessions than we were currently able to gather. We should keep in mind, nevertheless, that the current data was sufficiently reliable to classify rest from writing with $92 \%$ accuracy, and the employed classifications methods are sensitive enough to be used on smaller datasets. This forces us to look into alternative explanations as well before upscaling. One such explanation is that for this particular writer, the writing process itself may predominantly be a cognitive task and unrelated to the emotional content, i.e. the writer does not experience a particular emotion himself when writing about it. The neurophysiological pattern found in writing compared to rest and the facial expression (often classified as neutral) fit with 
517 the signature of a cognitive task. Based on the vast production of the writer and as confirmed in

518 later discussion with him, this is a viable option. In hindsight, the time pressure (2 minutes per

519 item), the strict instruction (write about this particular emotion fitting with this particular

520 picture), the time of day (always in the morning before the writing block started), and the

521 presence of the experimenter may all have triggered cognitive controlled creativity rather than

522 emotional or spontaneous creativity. The third factor that may have played a role in the current

523 results is the task setting that may have resulted in multiple processes (including but not limited

524 to emotional, associative, creative, linguistic and motor planning processes). The resulting brain

525 activity patterns may not be comparable to those for passive viewing of emotional pictures in a

526 laboratory environment.

\section{4.3.2 Subjective ratings}

528 The ratings of arousal, happy, optimistic and flow seem to show the same pattern. At the start of

529 the day, the writer is in a 'relaxed, good mood' but his mood seems to dwindle during the writing

530 with increasing arousal. At the end of the day, after the last writing session, this pattern stabilizes

531 or is reversed. This profile in part reflects the circadian modulation of mood and related aspects.

\section{The ebook of the future}

533 One may ask if uncovering brain states associated with art will de-mythologize the process: will

534 art lose its meaning, beauty or purity when reduced to activity of groups of neurons? Will we

535 eventually reveal the mechanisms of art and thus render it mechanical? Will scientists be able to

536 develop a drug that makes everyone a best-selling author? Will this knowledge increase the

537 'creativity rat race' for artistic and creative success as cognitive enhancers may do in the

538 'cognitive rat race' in the academic world [85]? We think not - but raising and discussing these 
539 questions is of utmost importance for the field [58]. A more interesting debate is whether

540 creative writing is a skill one can develop like skilled behavior in sports and music, or possibly

541 even non-creative writing like scientists and journalists do on a daily basis. Creative skills are

542 important outside the arts and the creative industry and their importance is widely acknowledged

543 in an innovative and knowledge-based economy. We would like to expand our research into

544 (spontaneous) creativity to answer important questions and develop appropriate tests and tools to 545 measure spontaneous creativity (which may require 24 hour measurements).

546 Current ebooks have the ability to track reader behavior and ebook retailers are actively

547 gathering (anonymous) data of their readers on parameters such as the books the reader has

548 finished (or not), how fast, where reading was discontinued and for how long and which words

549 were looked up in a connected dictionary [86]. None of this information is directly used for the

550 benefit of the reader but serves manufacturers and publishers only. The basis for our approach is

551 to measure the readers' state and behavior to make them the primary beneficiaries, for instance

552 through enhancing the reader experience. There are many approaches foreseeable. A relatively

553 simple one that is not interactive yet is to use the emotional response to give better informed

554 advice on other books the reader may enjoy. In a similar way, readers may want to share their

555 emotional profile, for instance by posting it on social media or through new communities of

556 people with similar frames of mind around a specific book. Real interactivity may also come in

557 many forms. For instance, the emotional response may be used to add music or other

558 multisensory stimuli to further intensify the experience or ultimately change the storyline or the

559 flow of the book. This may lead to new media products that are somewhere in between literature,

560 movies and games.

561 Acknowledgments 
562 We kindly acknowledge the great help of Christian Vermorken and Marc Grootjen from

563 Eaglescience, Andrew Spink from Noldus IT, and Leo Hoogendoorn from TMSi for providing

564 hardware and software components and helping us with the measurements and analyses. We

565 sincerely thank Arnon Grunberg and his publishers Elik Lettinga and Paulien Loerts from Nijgh

566 \& Van Ditmar for sharing their time and their creative minds.

\section{References}

568 [1] Grunberg, A. (2013). Merit. http://www.arnongrunberg.com/blog/2581-merit.

569 [2] He, Q., Xue, G., Chen, C., Chen, C., Lu, Z.-L., Dong, Q. Decoding the neuroanatomical basis

570

571

572

573

574

575

576

577

578

579

580

581

582

583

584

585

586

587

588

589

590

591 of reading ability: A multivoxel morphometric study (2013) Journal of Neuroscience, 33 (31), pp. 12835-12843.

[3] Carreiras, M., Armstrong, B.C., Perea, M., Frost, R. The what, when, where, and how of visual word recognition (2014) Trends in Cognitive Sciences, 18 (2), pp. 90-98.

[4] Nijhof AD, Willems RM (2015) SimulatingFiction: Individual Differences in LiteratureComprehension Revealed with fMRI. PLoS ONE10(2): e0116492.

[5] Dehaene, S., Cohen, L., Morais, J. \& Kolinsky, R. (2015). Illiterate to literate: behavioural and cerebral changes induced by reading acquisition. Nature Reviews Neuroscience, 16, 234-244.

[6] Berns, G.S., Blaine, K., Prietula, M.J., Pye, B.E. Short- and long-term effects of a novel on connectivity in the brain (2013) Brain Connectivity, 3 (6), pp. 590-600.

[7] Bal, P.M., Veltkamp, M. How Does Fiction Reading Influence Empathy? An Experimental Investigation on the Role of Emotional Transportation (2013) PLoS ONE, 8 (1), art. no. e55341.

[8] Johnson, D.R. Transportation into a story increases empathy, prosocial behavior, and perceptual bias toward fearful expressions (2012) Personality and Individual Differences, 52 (2), pp. 150-155.

[9] Kidd, D.C., Castano, E. Reading literary fiction improves theory of mind (2013) Science, 342 (6156), pp. 377-380.

[10] Hsu, C.-T., Conrad, M., Jacobs, A.M. Fiction feelings in Harry Potter: Haemodynamic response in the mid-cingulate cortex correlates with immersive reading experience (2014) NeuroReport, 25 (17), pp. 1356-1361. 
592 [11] Walter, H. Social cognitive neuroscience of empathy: Concepts, circuits, and genes (2012)

593

594

595

596

597

598

599

600

601

602

603

604

605

606

607

608

609

610

611

612

613

614

615

616

617

618

619

620

621

Emotion Review, 4 (1), pp. 9-17.

[12] Johansen, J.D. Feelings in literature (2010) Integrative Psychological and Behavioral Science, 44 (3), pp. 185-196.

[13] Mar, R.A., Oatley, K., Djikic, M., Mullin, J. Emotion and narrative fiction: Interactive influences before, during, and after reading (2011) Cognition and Emotion, 25 (5), pp. 818-833.

[14] Brouwer, A.-M., Hogervorst, M. Reuderink, B., Van der Werf, Y. \& Van Erp, J.B.F. (2015). Physiological signals distinguish between reading emotional and non-emotional sections in a novel. Brain-Computer Interfaces, 2 (2-3), 76-89.

[15] Ishizu, T., Zeki, S. The brain's specialized systems for aesthetic and perceptual judgment (2013) European Journal of Neuroscience, 37 (9), pp. 1413-1420.

[16] Kawabata, H., Zeki, S. Neural Correlates of Beauty (2004) Journal of Neurophysiology, 91 (4), pp. 1699-1705.

[17] Hasson, U., Landesman, O.,Knappmeyer, B., Vallines, I., Rubin, N., Heeger, D.J. (2008). Neurocinematics: The Neuroscience of Film. Projections, 2 (1), 1-26.

[18] Fleureau, J., Guillotel, P., Orlac, I. Affective benchmarking of movies based on the physiological responses of a real audience (2013) Proceedings - 2013 Humaine Association Conference on Affective Computing and Intelligent Interaction, ACII 2013, art. no. 6681410, pp. 73-77.

[19] Golland, Y., Keissar, K., Levit-Binnun, N. Studying the dynamics of autonomic activity during emotional experience (2015) Psychophysiology, 51 (11), pp. 1101-1111.

[20] Van der Werf, Y.D., Van Erp, J.B.F. Monitoring the Physiology of the Creative Process. In: A.J. Spink, L.W.S. Loijens, M. Woloszynowska-Fraser \& L.P.J.J. Noldus: Proceedings of Measuring Behavior 2014, (Wageningen, The Netherlands, August 27-29, 2014).

[21] Dalgleish, T. (2004). The emotional brain. Nature Reviews Neuroscience, 5(7), 582-589.

[22] Tovote, P., Fadok, J.P. \& Luthi, A. (2015). Neural circuits for fear and anxiety. Nature Reviews Neuroscience, 16 (6), 317-331.

[23] Isotani, T. Lehmann, D. Pascual-Marqui, R.D., Fukushima, M., Saito, N., Yagyu, T., Kinoshita, T. Source localization of brain electric activity during positive, neutral and 
negative emotional states, International Congress Series, Volume 1232, April 2002,

623 Pages 165-173.

624 [24] Ekman, P. Are there basic emotions? (1992) Psychological Review, 99 (3), pp. 550-553.

625 [25] Russell, J. (1980). A circumplex model of affect. Journal of Personality and Social 626 Psychology, 39, 1161-1178.

627 [26] Barrett, L.F., Russell, J.A. The structure of current affect: Controversies and emerging 628 consensus (1999) Current Directions in Psychological Science, 8 (1), pp. 10-14. 629 [27] Mauss, I.B., Robinson, M.D. Measures of emotion: A review (2009) Cognition and Emotion, 23 (2), pp. 209-237

631 [28] Russell, J.A., Barrett, L.F. Core affect, prototypical emotional episodes, and other things called emotion: Dissecting the elephant (1999) Journal of Personality and Social Psychology, 76 (5), pp. 805-819.

634 [29] Posner, J., Russell, J.A., Peterson, B.S. The circumplex model of affect: An integrative approach to affective neuroscience, cognitive development, and psychopathology (2005) Development and Psychopathology, 17 (3), pp. 715-734

[30] Rellecke, J., Palazova, M., Sommer, W., Schacht, A. On the automaticity of emotion processing in words and faces: Event-related brain potentials evidence from a superficial task (2011) Brain and Cognition, 77 (1), pp. 23-32.

[31] Colibazzi, T., Posner, J., Wang, Z., Gorman, D., Gerber, A., Yu, S. , Zhu, H., Kangarlu, A., arousal during the experience of induced emotion. Emotion, 10, 377-389.

[32] Herbert, C., Ethofer, T., Anders, S., Junghöfer, M., Wildgruber, D., Grodd, W. \& Kissler, J. (2009). Amygdala activation during reading of emotional adjectives - An advantage for pleasant content. Social Cognitive and Affective Neuroscience, 4, 35-49.

647

[33] Kuchinke, L., Jacobs, A. M., Gubrich, C., Võ, M. L.-H., Conrad, M., \& Herrmann, M. (2005). Incidental effects of emotional valence in single word processing: An fMRI study. NeuroImage, 28, 1022-1032.

[34] Lewis, P. A., Critchley, H. D., Rotshtein, P., \& Dolan, R. J. (2007). Neural correlates of processing valence and arousal in affective words. Cerebral Cortex, 17, 742-748. 
653

654

655

656

657

658

659

660

661

662

663

664

665

666

667

668

669

670

671

672

673

674

675

676

677

678

679

680

681

682

683

An fMRI study of the affective circumplex using emotion-denoting words. Human Brain Mapping, 30, 883-895.

[36] Kissler, J., Assadollahi, R., \& Herbert, C. (2006). Emotional and semantic networks in visual word processing: Insights from ERP studies. Progress in Brain Research, 156, $147-183$.

[37] Citron, F.M.M. (2012). Neural correlates of written emotion word processing: A review of recent electrophysiological and hemodynamic neuroimaging studies. Brain \& Language, 122, 211-226.

[38] Min, Y.-K., Chung, S.-C., Min, B.-C. Physiological evaluation on emotional change induced by imagination (2005) Applied Psychophysiology Biofeedback, 30 (2), pp. 137150

[39] Bayer, M., Sommer, W., Schacht, A. Reading emotional words within sentences: The impact of arousal and valence on event-related potentials (2010) International Journal of Psychophysiology, 78 (3), pp. 299-307.

[40] Herbert, C., Junghofer, M., Kissler, J. Event related potentials to emotional adjectives during reading (2008) Psychophysiology, 45 (3), pp. 487-498

[41] Holt, D.J., Lynn, S.K., Kuperberg, G.R. Neurophysiological correlates of comprehending emotional meaning in context (2009) Journal of Cognitive Neuroscience, 21 (11), pp. 2245-2262.

[42] Bahramisharif, A., van Gerven, M., Heskes, T., Jensen, O.: Covert attention allows for continuous control of brain-computer interfaces. European Journal of Neuroscience 31(8), 1501-1508 (2010).

[43] Klimesch, W., Sauseng, P., Hanslmayr, S.: EEG alpha oscillations: the inhibition-timing hypothesis. Brain Res. Rev. 53, 63-88 (2007)

[44] Ko, K.-E., Yang, H.-C., Sim, K.-B. Emotion recognition using EEG signals with relative power values and Bayesian network (2009) International Journal of Control, Automation and Systems, 7 (5), pp. 865-870.

[45] Fox, N.A. If it's not left: It's right electroencephalograph asymmetry and the development of emotion (1991) American Psychologist, 46 (8), pp. 863-872.

[46] Schmidt, L.A., Trainor, L.J. Frontal brain electrical activity (EEG) distinguishes valence and intensity of musical emotions (2001) Cognition and Emotion, 15 (4), pp. 487-500. 
684 [47] Tomarken, A.J., Davidson, R.J., Wheeler, R.E., Doss, R.C. Individual Differences in

685

686

687

688

689

690

691

692

693

694

695

696

697

698

699

700

701

702

703

704

705

706

707

708

709

710

711

712

Anterior Brain Asymmetry and Fundamental Dimensions of Emotion (1992) Journal of Personality and Social Psychology, 62 (4), pp. 676-687.

[48] Lewis, R.S., Weekes, N.Y., \& Wang, T.H. (2007). The effect of a naturalistic stressor on frontal EEG asymmetry, stress, and health. Biological Psychology, 75 (3), 239-247.

[49] Verona, E., Sadeh, N., \& Curtin, J.J. (2009). Stress-Induced Asymmetric Frontal Brain Activity and Aggression Risk. Journal of Abnormal Psychology, 118 (1), 131-145.

[50] Brouwer, A.-M., Hogervorst, M. A., Herman, P., \& Kooi, F. (2009). Are You Really Looking? Finding the Answer through Fixation Patterns and EEG. Lecture Notes In Artificial Intelligence, Vol. 5638: Proceedings of the 5th International Conference on Foundations of Augmented Cognition, 329-338.

[51] Muehl, C., Van Den Broek, E., Brouwer, A.-M., Nijboer, F., van Wouwe, N., \& Dirk Heylen, D. (2011). Multi-modal Affect Induction for Affective Brain-Computer Interfaces. Affective Computing and Intelligent Interaction - Lecture Notes in Computer Science, 2011, Volume 6974/2011, 235-245

[52] Roth, W. T. (1983). A comparison of P300 and the skin conductance response. In A. W. K. Gaillard and W. Ritter (Eds.), Tutorials in ERP research—endogenous components (pp. 177-199). Amsterdam: North-Holland.

[53] Berntson, G.G., Bigger, J.T., Eckberg, D.L., Grossman, P., Kaufmann, P.G., Malik, M., Nagaraja, H.N., Porges, S.W., Saul, J.P., Stone, P.H., and Van der Molen, M.W., (1997). Heart rate variability: origins, methods, and interpretive caveats. Psychophysiology, 34(6), 623-648.

[54] Kreibig, S.D. Autonomic nervous system activity in emotion: A review. Biological Psychology, vol. 84, no. 3. pp.394-421, 2010.

[55] Van Erp, J.B.F., Brouwer, A.-M. \& zander, T.O. (2015). Editorial: Using neurophysiological signals that reflect cognitive or affective state. Frontiers in Neuroscience, 9:00193.

[56] Dockray, S. and Steptoe, A. Positive affect and psychobiological processes. Neuroscience \& Biobehavioral Reviews, vol. 35, no. 1. pp.69-75, Sept., 2010. 
713 [57] Brouwer, A.-M., Zander, T.O., Van Erp, J.B.F., Korteling, H., Bronkhorst, A.W. Using

714

715

716

717

718

719

720

721

722

723

724

725

726

727

728

729

730

731

732

733

734

735

736

737

738

739

740

741

742 neurophysiological signals that reflect cognitive or affective state: Six recommendations to avoid common pitfalls. Frontiers in Neuroscience, 9:00136.

[58] Van Erp, J.B.F., Lotte, F. \& Tangermann, M. (2012). Brain-Computer Interfaces: Beyond Medical Applications. IEEE computer, 45(4), 26-34.

[59] Van Erp, J.B.F.; Brouwer, A.-M.; Thurlings, M.E. \& Werkhoven, P.J. (2013). Framework for BCIs in Multimodal Interaction and Multitask Environments, Towards Practical BrainComputer Interfaces. Pp. 239-250, 2013, Springer Berlin Heidelberg.

[60] Van Erp, J.B.F.; Thurlings, M.E.; Brouwer, A.-M. \& Werkhoven, P.J. (2011). BCIs in multimodal interaction and multitask environments: theoretical issues and initial guidelines,Universal Access in Human-Computer Interaction. Users Diversity, pp 610619. Springer Berlin Heidelberg.

[61] Reuderink, B. and Mühl, C. and Poel, M. (2013) Valence, arousal and dominance in the EEG during game play. International Journal of Autonomous and Adaptive Communications Systems, 6 (1). pp. 4562.ISSN 17548632

[62] Leslie, Grace and Ojeda, Alejandro and Makeig, Scott. Measuring musical engagement using expressive movement and EEG brain dynamics. Psychomusicology: Music, Mind, and Brain, Volume 24, number 1, Pages 75-91, 2014

[63] Piffer, D. (2012). Can creativity be measured? An attempt to clarify the notion of creativity and general directions for future research. Thinking skills and Creativity, 7, 258-264.

[64] Dietrich, A., Kanso, R. A review of EEG, ERP, and neuroimaging studies of creativity and insight (2010) Psychological Bulletin, 136 (5), pp. 822-848

[65] Arden, R., Chavez, R.S., Grazioplene, R., Jung, R.E. Neuroimaging creativity: A psychometric view (2010) Behavioural Brain Research, 214 (2), pp. 143-156.

[66] Dietrich, A. The cognitive neuroscience of creativity (2004) Psychonomic Bulletin and Review, 11 (6), pp. 1011-1026.

[67] Ellamil, M., Dobson, C., Beeman, M., Christoff, K. Evaluative and generative modes of thought during the creative process (2012) NeuroImage, 59 (2), pp. 1783-1794

[68] Limb, C.J., Braun, A.R.Neural substrates of spontaneous musical performance: An fMRI study of jazz improvisation(2008) PLoS ONE, 3 (2), art. no. e1679 
743 [69] Liu, S., Chow, H.M., Xu, Y., Erkkinen, M.G., Swett, K.E., Eagle, M.W., Rizik-Baer, D.A.,

744

745

746

747

748

749

750

751

752

753

754

755

756

757

758

759

760

761

762

763

764

765

766

767

768

769

770

771

772

773 Braun, A.R.Neural correlates of lyrical improvisation: An fMRI study of freestyle $\operatorname{rap}(2012)$ Scientific Reports, 2, art. no. 834

[70] Fink, A., Benedek, M. EEG alpha power and creative ideation (2014) Neuroscience and Biobehavioral Reviews, 44, pp. 111-123.

[71] Mihov, K.M., Denzler, M., Förster, J. Hemispheric specialization and creative thinking: A meta-analytic review of lateralization of creativity (2010) Brain and Cognition, 72 (3), pp. 442-448.

[72] Mayseless, N., Aharon-Peretz, J., Shamay-Tsoory, S. Unleashing creativity: The role of left temporoparietal regions in evaluating and inhibiting the generation of creative ideas (2014) Neuropsychologia, 64, pp. 157-168

[73] Shamay-Tsoory, S.G., Adler, N., Aharon-Peretz, J., Perry, D., Mayseless, N. The origins of originality: The neural bases of creative thinking and originality (2011) Neuropsychologia, 49 (2), pp. 178-185.

[74] Carlsson, I., Wendt, P.E., Risberg, J. On the neurobiology of creativity. Differences in frontal activity between high and low creative subjects (2000) Neuropsychologia, 38 (6), pp. $873-885$.

[75] Chávez-Eakle, R.A., Graff-Guerrero, A., García-Reyna, J.-C., Vaugier, V., Cruz-Fuentes, C. Cerebral blood flow associated with creative performance: A comparative study (2007) NeuroImage, 38 (3), pp. 519-528.

[76] Shah, C., Erhard, K., Ortheil, H.-J., Kaza, E., Kessler, C., Lotze, M. Neural correlates of creative writing: An fMRI Study (2013) Human Brain Mapping, 34 (5), pp. 1088-1101.

[77] Erhard, K., Kessler, F., Neumann, N., Ortheil, H.-J., Lotze, M. (2014). Professional training in creative writing is associated with enhanced fronto-striatal activity in a literary text continuation task. NeuroImage, 100, 15-23.

[78] Lotze, M., Erhard, K., Neumann, N., Eickhoff, S.B., Langner, R. (2014). Neural correlates of verbal creativity: Differences in resting-state functional connectivity associated with expertise in creative writing. Frontiers in Human Neuroscience, 8, 00516.

[79] Lang, P. J., Bradley, M. M., \& Cuthbert, B. N. (1999). International affective picture system (IAPS): Instruction manual and affective ratings. The center for research in psychophysiology, University of Florida. 
774 [80] Russell, J.A., Weiss, A., Mendelsohn, G.A. Affect Grid: A Single-Item Scale of Pleasure

775

776

777

778

779

780

781

782

783

784

785

786

787

788

789

790

791

792

793 and Arousal (1989) Journal of Personality and Social Psychology, 57 (3), pp. 493-502

[81] Fredrickson, B.L., Tugade, M.M., Waugh, C.E., Larkin, G.R. What Good Are Positive Emotions in Crises? A Prospective Study of Resilience and Emotions Following the Terrorist attacks on the United States on September 11th, 2001 (2003) Journal of Personality and Social Psychology, 84 (2), pp. 365-376.

[82] van Gerven, M., Bahramisharif, A., Farquhar, J., and Heskes, T. (2013). Donders Machine Learning Toolbox (DMLT) for Matlab Version 26/06/2013. Available online at: https://github.com/distrep/DMLT.

[83] Friedman, J., Hastie, T., and Tibshirani, R. (2010). Regularization paths for generalized linear models via coordinate descent. J. Stat. Softw. 33, 1-22.

[84] Lemm, S., Blankertz, B. Dickhaus, T. Müller, K.R. Introduction to machine learning for brain imaging. Neuroimage 56 (2), 387-399

[85] Repantis, D. Schlattmann, P. Laisney, O. \& Heuser, I. Modafinil and methylphenidate for neuroenhancement in healthy individuals: A systematic review, Pharmacological Research 62, 187-206 (2010).

[86] Flood, A. (2014). Ebooks can tell which novels you didn't finish. http://www.theguardian.com/books/2014/dec/10/kobo-survey-books-readers-finishdonna-tartt. 
794 Figure captions

795 Figure 1. Location of the 28 electrodes in the 10-20 system.

796 Figure 2. Writer showing the neurophysiological sensors (A) and during writing (B).

797 Figure 3. Results of the physiological measures Heart Rate (A) and rmssd RRI (B) as function of 798 the different calibration blocks. Eyes open and Eyes closed blocks were measured before 799 and after the emotional blocks. The order of the emotional blocks was balanced over 800 days. Error bars denote the standard error of the mean.

801 Figure 4. The left column shows the power in the different frequency bands in the rest blocks 802 (eyes open and eyes closed combined) and the middle column in the writing blocks. The 803 right column gives the weights of the features in the classification model.

804 Figure 5. Significant changes in subjective ratings over the course of a writing day.

805

806 Table captions

807 Table 1. Experimental protocol for one day.

808 Table 2. Specification of the experimental protocol. 